\title{
Melhoria Contínua de Processo de Software Utilizando a Teoria das Restrições
}

\author{
Thiago Costa ${ }^{1}$, Ana Regina C. Rocha ${ }^{1}$, Gleison Santos ${ }^{2}$ \\ ${ }^{1}$ COPPE/UFRJ - Universidade Federal do Rio de Janeiro \\ Programa de Engenharia de Sistemas e Computação \\ Av. Horácio Macedo, 2030, Prédio do Centro de Tecnologia, Bloco H, Sala 319 Caixa \\ Postal 68511 - CEP 21941-914 - Rio de Janeiro, RJ \\ ${ }^{2}$ Programa de Pós-Graduação em Informática - Universidade Federal do Estado do Rio \\ de Janeiro (UNIRIO) - Av. Pasteur, 458, Urca, CEP 22290-240 - Rio de Janeiro, RJ \\ \{thiagom, darocha\}@cos.ufrj.br, gleison.santos@uniriotec.br
}

\begin{abstract}
The software development process is composed by elements that interact to produce intermediate and final products and may cause effects that influences desirably or undesirably its performance. The proposed approach for continuous software process improvement systematizes the investigation of factors that cause undesirable effects in order to identify and remove the factor that most influences undesirably the process performance. To do so, the approach applies the concepts of Thinking Processes of the Theory of Constraints. An empirical study was performed by applying the approach into a specific scenario in the industry resulting in an improvement proposal to this process. Evidence of suitability and viability were observed in this study.
\end{abstract}

Resumo. O processo de desenvolvimento de software é composto por elementos que, ao interagirem para produzir os produtos intermediários e finais, ocasionam efeitos que podem influenciar de maneira desejável ou indesejável o seu desempenho. A abordagem proposta para melhoria contínua de processo de software sistematiza a investigação de fatores que causam efeitos indesejáveis, a fim de identificar e remover o fator que mais influencia o desempenho do processo de maneira indesejada. Para isso, são aplicados os conceitos dos Processos de Raciocínio da Teoria das Restrições. Um estudo experimental foi executado, aplicando a abordagem em um cenário especifico na indústria, resultando em uma proposta de melhoria para esse processo. Indícios de adequabilidade e viabilidade foram observados neste estudo.

\section{Introdução}

O processo de desenvolvimento de software é composto por elementos que estão envolvidos durante a sua execução. Insumos, procedimentos, ferramentas e pessoas são exemplos de elementos do processo de desenvolvimento que, ao interagirem para produzir os produtos intermediários e produtos finais, ocasionam efeitos que podem influenciar de maneira desejável ou indesejável o desempenho do processo.

No contexto de melhoria contínua de processo, o desempenho do processo deve ser monitorado, a fim de identificar causas e fatores que possam servir para identificar oportunidades de melhoria e para elaborar soluções. Dessa forma, ao considerar que esses fatores de influência estão presentes nos processos de software, é importante 
maximizar as chances de identificar as oportunidades de melhoria que mais podem contribuir para a melhoria no desempenho do processo antes da implantação em toda a empresa. Além disso, é importante apoiar os executores da melhoria na elaboração da solução, minimizando os efeitos indesejáveis que tal modificação pode causar no desempenho do processo. Esta análise, no entanto, não é trivial, uma vez que a melhoria do desempenho dos processos de software envolve diversos fatores de influência que estão inter-relacionados.

Este artigo apresenta uma abordagem para melhoria de processo de software utilizando a Teoria das Restrições (TR) (Goldratt 1990) através dos seus Processos de Raciocínio (PR) (Scheinkopf 1999). Os Processos de Raciocínio implementam uma abordagem para melhoria contínua de processos que sistematiza o raciocínio utilizado para identificar oportunidades de melhoria que mais impactam indesejavelmente o desempenho do processo e para elaborar soluções para tais oportunidades. A Teoria das Restrições preconiza que, analogamente a uma corrente, o desempenho do processo possui um elo mais fraco. Dessa forma, o desempenho do processo pode ser melhorado continuamente de maneira mais eficaz ao se concentrar os esforços de melhoria sobre o seu elo mais fraco, identificando e removendo os fatores de influência indesejáveis que mais impactam negativamente o desempenho do processo. Além da abordagem, este artigo descreve o estudo experimental executado no contexto de sua definição para avaliação da sua aplicação em um cenário na indústria. A definição do estudo e as condições de execução e seus resultados são apresentados, caracterizando a empresa e os participantes, assim como exemplos de produtos resultante da aplicação dos PR. O estudo aponta indícios de adequabilidade e viabilidade para a aplicação dos PR para a melhoria de processo de software.

Além desta introdução, este artigo está organizando em outras 5 seções. $\mathrm{Na}$ segunda seção, os conceitos de melhoria contínua de processo de software e da Teoria das Restrições são explicados para contextualizar a abordagem proposta. Na seção 3, a abordagem é apresentada. Na seção 4, o estudo experimental executado e seus resultados são descritos. Por fim, na seção 5, as considerações finais são apresentadas, assim como as contribuições e perspectivas futuras.

\section{Melhoria Contínua de Processo de Software e a Teoria das Restrições}

O desenvolvimento de software é uma tarefa complexa e influenciada por fatores técnicos, culturais e ambientais. As atividades de desenvolvimento de software se diferenciam por serem baseadas intensivamente no esforço humano e colaborativo; e por produzirem, na maioria das vezes, produtos diferentes a cada execução (Lindvall e Rus 2000).

A melhoria do processo de software tem se estabelecido nas organizações que perseguem uma melhor competitividade (Montoni 2010). A melhoria contínua de processo de software permite que, sistematicamente, as organizações alcancem melhores índices de produtividade e qualidade. O conceito de ciclo de melhoria foi introduzido por Deming e seu conceitos de Gestão da Qualidade Total (TQM - Total Quality Management), e que estão presentes atualmente em normas e modelos de processo de software, tais como, ISO/IEC 15504 (ISO/IEC 2003), ISO/IEC 12207 (ISO/IEC 2008), MR-MPS-SW (SOFTEX 2012) e CMMI (SEI 2010). De acordo com essas abordagens, o ciclo contínuo de melhoria dos processos de software deve englobar 
uma estratégia que considere diferentes fontes de oportunidades de melhoria para tornar os processos mais eficientes e capazes.

Um mapeamento sistemático foi desenvolvido no contexto da definição da abordagem para levantar quais abordagens propõem a identificação de oportunidade de melhorias em organizações de software que implementam a melhoria contínua, focando a identificação de abordagens que sistematizassem ou apoiassem a melhoria contínua em organizações de software de alta maturidade ou que utilizassem métodos quantitativos para gerenciar e melhorar o desempenho de seus processos. O protocolo, execução e resultados do estudo estão descritos em (Costa 2012),

Diversas abordagens identificadas no mapeamento, além das normas e modelos supracitados, definem técnicas estatísticas para apoiar a melhoria contínua de processos, tais como, o Controle Estatístico de Processo (CEP) e o Seis Sigma. Alguns trabalhos descrevem a aplicação dessas abordagens para implementar uma metodologia de melhoria contínua em processos de software, como, por exemplo, a aplicação do Seis Sigma para Qualidade de Software (Hong e Goh 2003) ou Controle Estatístico de Processo para Melhoria de Processo de Software (Florac e Carleton 1999).

Tais trabalhos descrevem modelos de referências, técnicas, processos e arcabouços para melhoria dos processos, sem, no entanto, descrever como atingir a melhoria contínua. O resultado do mapeamento sistemático permitiu identificar lacunas que tais abordagens não preenchem. O CEP, por exemplo, embora defina de que forma as técnicas estatísticas devem ser utilizadas para implementar o controle estatístico de processo, apontando variações no desempenho do processo, não descreve, no entanto, como identificar a causa dessas variações e como eliminá-las em um ciclo de melhoria contínua.

A Teoria das Restrições é uma metodologia de melhoria contínua desenvolvida inicialmente na manufatura e que, assim como a abordagem de melhoria contínua de Deming (PDCA) e demais abordagens de TQM, objetiva melhorar continuamente processos de maneira estruturada e sistemática. A TR preconiza que a limitação do desempenho do processo é ocasionada por algumas poucas restrições (normalmente uma) que, se removidas de maneira sistemática, podem resultar em ganhos mais consideráveis para o desempenho do processo (Scheinkopf 1999). Os Processos de Raciocínio são as ferramentas da TR para implementar a melhoria contínua nos processo, sendo compostos por um conjunto de fundamentos lógicos e elementos gráficos que são utilizados para construir os seus diagramas.

De acordo com a TR, uma restrição é definida como qualquer fator ou efeito que limite o alcance de um melhor desempenho do processo em relação ao seu objetivo de desempenho. As restrições são classificadas em três categorias principais: físicas, políticas e comportamentais (Scheinkopf 1999). Existem restrições de todas as três categorias em qualquer processo, a qualquer momento, e estas estão relacionadas. Restrições comportamentais são resultados de paradigmas e comportamentos que resultam em restrições políticas que, por sua vez, causam restrições físicas. Restrições comportamentais e políticas são normalmente mais difíceis de serem removidas e suas remoções são as que têm potencial de maior ganho de desempenho para o processo.

De acordo com Scheinkopf (1999), um ponto forte da TR e seus Processos de Raciocínio é o mapeamento do processo sob o ponto de vista do problema a ser resolvido. Dessa forma, apenas aspectos considerados pertinentes ao desempenho 
indesejado do processo são mapeados, permitindo que problemas complexos sejam resolvidos sem recorrer à representação completa de todos os fatores que influenciam o processo.

Os diagramas são resultado da aplicação dos Processos de Raciocínio e são compostos por quatro árvores e um diagrama em formato de nuvem, a saber: (i) Árvore da Realidade Atual (ARA); (ii) Diagrama de Resolução de Conflito (DRC); (iii) Árvore da Realidade Futura (ARF); (iv) Árvore de Pré-Requisitos (APR) e (v) Árvore de Transição (ATR). Os Processos de Raciocínio são aplicados para responder as seguintes questões de mudanças: i) o que mudar; ii) para o que mudar; e iii) como causar a mudança. A correspondência e ordem da aplicação dos PR para responder às questões de mudança são representadas na Tabela 1 .

Tabela 1 - Aplicação dos Processos de Raciocínio em relação às questões de mudança (Scheinkopf, 1999)

\begin{tabular}{|c|l|}
\hline O que mudar? & 1. Árvore da Realidade Atual \\
\hline Para o que mudar? & 2. Diagrama de Resolução de Conflitos \\
\hline & 3. Árvore da Realidade Futura \\
\hline 4. Árvore de Pré-requisitos \\
\hline Como causar a mudança? & 5. Árvore de Transição \\
\hline
\end{tabular}

Vários aspectos da TR e seus PR apresentam, em suas principais características, indícios de aplicabilidade à melhoria de processos de software, tais como: i) similaridades e integração com outras metodologias de TQM que já foram aplicadas à melhoria de processo de software; ii) regras lógicas para construção dos diagramas que permitam mapear os diversos aspectos e fatores de influência inerentes aos processos de software responsáveis pelas variações especiais e variações comuns ${ }^{1}$; iii) foco na eliminação de restrições comportamentais e políticas como principais limitantes do desempenho de sistemas e processos; iv) apoio à institucionalização de mudanças e melhorias no contexto organizacional de maneira sistemática e cíclica.

Ademais, alguns trabalhos têm considerado similaridades entre a TQM e a TR (Gupta e Boyd 2008). Neste contexto, pesquisas foram desenvolvidas propondo combinações entre a TR e outras abordagens de TQM que já foram aplicadas à melhoria de processo de software, tais como, Seis Sigma, Controle Estatístico de Processo, Lean Thinking e Teoria das Restrições (Lepore e Cohen 1999; Nave 2002; Davies, Mabin et al. 2004; Davies, Mabin et al. 2005; Ehie e Sheu 2005; Pirasteh e Farah 2006; Watson, Blackstone et al. 2007). Na seção seguinte é descrita a proposta de implementação de uma abordagem para melhoria contínua de processo de software utilizando a TR por meio dos seus PR.

\section{Proposta}

A abordagem apresentada neste artigo propõe a aplicação das ferramentas PR para a melhoria de processo de software. O fluxo das etapas do processo da abordagem

${ }^{1} \mathrm{O}$ desempenho de qualquer processo possui dois tipos de variação. Variações especiais causadas por problemas de execução (causas atribuíveis ou especiais) e variações comuns causadas pelas interações inerentes dos elementos que o compõem e pelas eventualidades de caráter natural ou ambiental (causas comuns) (Florac e Carleton 1999). 
proposta se baseia em abordagens de melhoria de processo de software identificadas na revisão da literatura, mais especificamente em trabalhos desenvolvidos no mesmo grupo de pesquisa (Albuquerque 2008; Campos 2008).

Por intermédio dos PR, a abordagem proposta permite analisar, de maneira mais abrangente, o processo em melhoria, ao mesmo tempo em que segue o rigor dos passos estruturados dos PR aplicados na identificação das oportunidades de melhoria, na elaboração da proposta de solução para tais oportunidades e na proposta de melhoria. Um esquema representando o fluxo de utilização dos PR no processo da abordagem e de seus principais elementos é ilustrado na Figura 1.

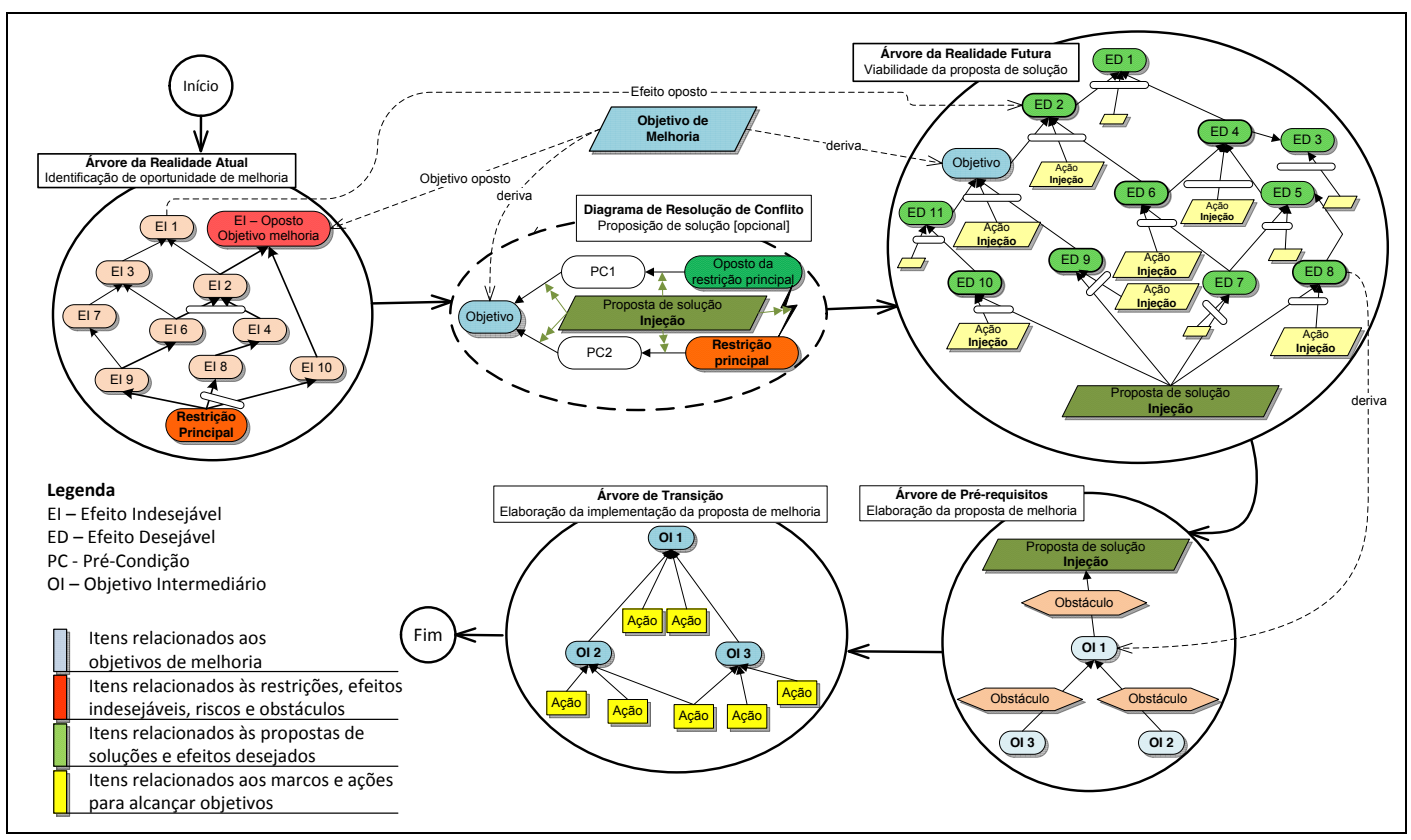

Figura 1 - Fluxo de utilização dos Processos de Raciocínio no processo da abordagem

A ordem da aplicação dos PR na Figura 1 acontece similarmente como descrita na Tabela 1. Embora os PR possam ser aplicados independentemente um do outro, a sua aplicação em conjunto permite que o processo de melhoria de processo: i) identifique a oportunidade de melhoria (principal restrição) por meio da análise dos efeitos indesejáveis da ARA; ii) apoie a elaboração da proposta de solução, resolvendo conflitos, caso haja, utilizando o DRC; iii) averigue a viabilidade da proposta de solução, identificando quais as possíveis ações que podem ser tomadas para atingir efeitos desejáveis que resultem no objetivo da proposta de solução através da ARF; iv) auxilie na elaboração da proposta de melhoria, definindo objetivos intermediários e riscos envolvidos através da APR; e v) apoie no detalhamento das ações necessárias para implementar a proposta de melhoria, considerando seus objetivos intermediários, por meio da ATR.

Cada diagrama possui elementos específicos, tais como efeitos desejáveis (ED), efeitos indesejáveis (EI), pré-condições (PC), causas suficientes, injeções (possíveis ações injetadas na ARF), objetivos intermediários (OI), obstáculos entre outros. As descrições de cada elemento, das relações de causa e efeito e dos procedimentos das PR 
aplicados para o processo da abordagem proposta e da definição formal do processo podem ser encontradas com detalhes em (Costa 2012).

A Figura 1 apresenta também a relação que existe entre os diversos elementos de diagramas distintos, tais como: os efeitos indesejáveis identificados na ARA que são reformulados na ARF para refletirem efeitos desejáveis; ou os efeitos desejáveis da ARF que derivam objetivos intermediários na APR. Ademais, elementos similares (representados na Figura 1 por sombreamento/tonalidade semelhantes) podem aparecer em mais de um diagrama em momentos distintos. Por exemplo, ação injeção na ARF é uma suposição, enquanto ação na ATR é uma ação ou tarefa exequível. Na próxima subseção "Atividades do Processo", detalha-se a utilização dos principais elementos citados na Figura 1.

O processo da abordagem foi dividido para ser apresentado na Figura 2 e Figura 3, sendo composto por seis etapas sequenciais: (i) Definir objetivo de melhoria; (ii) Preparar para Análise; (iii) Identificar restrição; (iv) Elaborar proposta de melhoria; (v) Implementar a melhoria candidata; e (v) Registrar resultados.

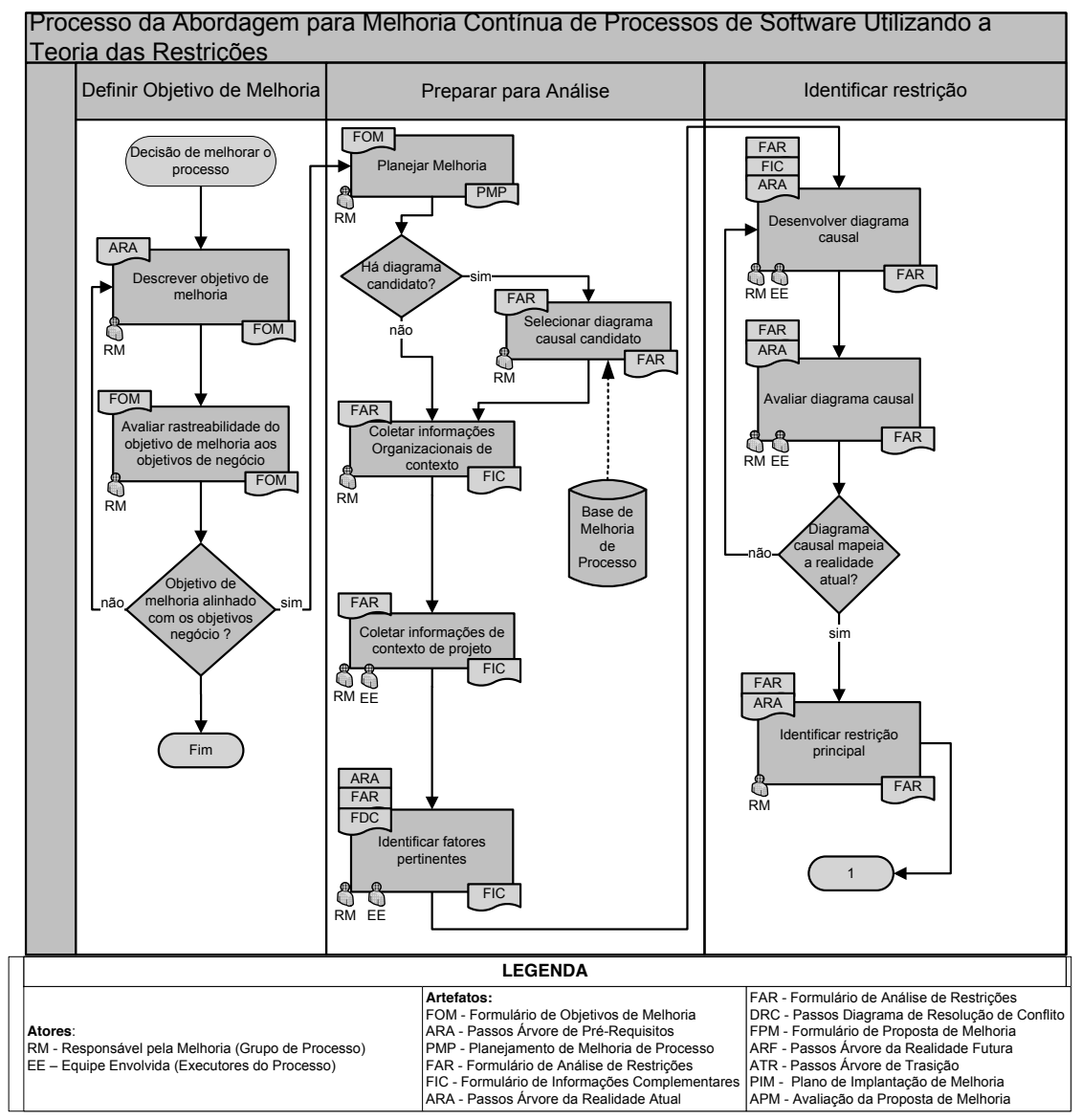

Figura 2 - Processo da abordagem proposta ( $\left(1^{\mathrm{a}}\right.$ parte)

Para utilizar o processo proposto, é necessário que a organização possua processos organizacionais definidos e que uma decisão de melhoria de determinado processo seja tomada pela alta direção ou departamento responsável. Embora a abordagem seja aplicável a qualquer processo definido, com base nos resultados do estudo experimental executado, sugere-se que seja utilizada em processos maduros, 
cujos problemas mais óbvios e fáceis de serem solucionados já foram removidos, justificando, assim, o rigor da aplicação da abordagem proposta.

A abordagem requer que um objetivo de melhoria seja definido no início. Isso se torna mais adequado quando se trata de processos cujos desempenhos são conhecidos quantitativamente, pois, desta forma, há possibilidade de estabelecer objetivos reais de melhoria e, consequentemente, meios de observar se houve melhoria nos desempenhos.

Além das características organizacionais citadas anteriormente, a participação de um Responsável do Grupo de Melhoria (RGP) - ou profissional que tenha competência para melhoria e definição de processo de software - e membros da equipe que executam o processo alvo da melhoria é imprescindível para a aplicação da abordagem. Formulários foram definidos para auxiliar a execução do processo e para servir como ativos de processo e raciocínios de decisões que deverão fazer parte da base de melhoria e de lições aprendidas da organização, mantendo, assim, o ciclo de melhoria contínua.

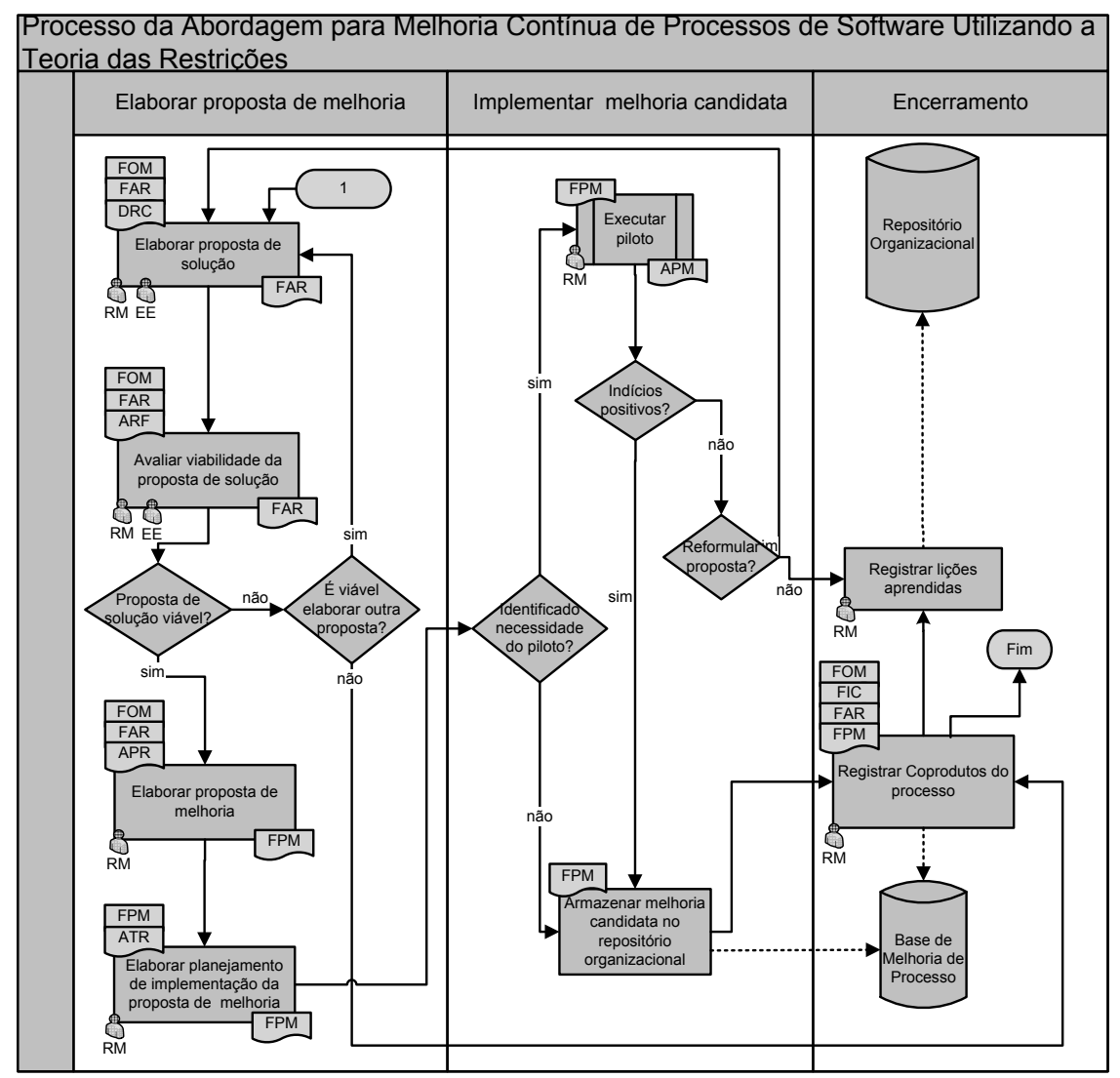

Figura 3 - Processo da abordagem proposta ( $2^{\mathrm{a}}$ parte $)$

\subsection{Atividades do Processo}

O início do processo de melhoria acontece após a decisão da organização de melhorar o processo organizacional. Neste momento, o objetivo de melhoria é definido de maneira estruturada e seu alinhamento com os objetivos da organização é averiguado. O objetivo de melhoria precisa estar definido e descrito de forma que todos os envolvidos na investigação da oportunidade de melhoria e proposição de solução tenham uma noção clara do objetivo a ser perseguido, assim como o escopo da investigação e da análise. 
A segunda atividade, "Preparar para análise", tem o objetivo de coletar e preparar os insumos para a identificação da restrição principal sobre a qual deve ser elaborada a proposta de solução. Além do planejamento da execução do processo de melhoria, neste momento o ciclo de melhoria é implementado por meio da recuperação de artefatos, análises e raciocínios utilizados em execuções anteriores para melhorar o mesmo processo. O levantamento das informações de contexto organizacionais e de projeto faz parte da aplicação da ARA. Um dos pontos positivos dessa atividade é o brainstorming para alinhamento do entendimento do objetivo de melhoria.

A atividade "Identificar restrição" tem o propósito de identificar a restrição principal que limita o desempenho do processo alvo da melhoria. As tarefas que compõem esta atividade aplicam os passos da ARA para construir o Diagrama Causal que representa os relacionamentos de causa e efeito entre os fatores de influência que resultam em efeitos indesejáveis. Os membros da equipe participam do escrutínio das causas e efeitos e avaliam se o diagrama representa a realidade atual com seus efeitos indesejáveis. A construção em conjunto do diagrama fomenta um consenso por parte dos membros da equipe sobre restrições ao desempenho do processo.

A atividade "Elaborar proposta de melhoria" tem como propósito elaborar uma proposta de solução para a restrição principal, avaliar a viabilidade da proposta de solução e elaborar a proposta de melhoria. De acordo com a TR, a proposta de solução pode ser a simples remoção da restrição principal. No entanto, há situações em que essa remoção não é trivial ou não pode acontecer. Para estes casos, opcionalmente, o processo proposto aplica o DRC para dirimir conflitos e atingir o objetivo de melhoria. A aplicação do DRC é positiva, pois auxilia na anulação da restrição principal, considerando os conflitos entre possibilidades de mudança e o objetivo da melhoria.

No segundo passo da atividade, uma avaliação da viabilidade da proposta de solução é realizada por meio da aplicação da ARF. A ARF reflete de maneira inversa os efeitos indesejáveis identificados pela ARA no Diagrama Causal, estabelecendo-os como efeitos desejáveis (condições futuras pressupostas) e apoiando-os por meio da identificação de ações (injeções) necessárias para sustentar tais efeitos. Neste momento são identificados os primeiros marcos, possíveis efeitos colaterais e ações de mitigação que devem ser tomadas para tornar a proposta de solução viável.

Se a proposta de solução for considerada viável, o terceiro passo da atividade propõe que seja elaborada a proposta de melhoria utilizando a APR. Os efeitos desejáveis pressupostos e os efeitos colaterais identificados na ARF são considerados, respectivamente, objetivos intermediários e obstáculos. Neste momento, a APR permite avaliar quais os riscos (obstáculos) de implementação da proposta de solução, identificando objetivos intermediários adicionais àqueles já identificados pela ARF. A perspectiva da APR para a melhoria de processo são as condições necessárias para que todos os riscos sejam superados, a fim de atingir o objetivo da proposta de solução.

O último passo da atividade é o detalhamento da proposta de melhoria no qual um plano de ações é gerado a partir da aplicação da ATR, definindo temporalmente a sequência das ações para atingir cada objetivo intermediário identificado na APR. A aplicação da ATR, além do plano de ações, gera insumos para a elaboração do cronograma, do orçamento, da identificação de riscos e ações de mitigação da proposta de melhoria. 
A atividade "Implementar melhoria candidata" é responsável no processo por avaliar a proposta de melhoria e verificar qual o resultado da execução de pilotos, quando necessário. Para isso, são aplicados os passos descritos na abordagem de (Silva Filho 2006) que propõe um processo para execução de projeto piloto voltado para organizações de software. Desta forma, se a proposta de melhoria tiver indícios de resultados positivos ao desempenho do processo, deve ser incluída na Base de Melhoria da organização para implementação em momento oportuno.

A última atividade "Registrar resultados" tem o objetivo de manter o ciclo de melhoria, alimentando a Base de Melhoria e de conhecimento da organização com os artefatos, análises e raciocínios produzido neste processo de melhoria, assim como suas lições aprendidas.

\section{Estudo Experimental}

O estudo experimental foi realizado em uma empresa de desenvolvimento de software. A empresa, com sede no estado do Rio de Janeiro, era certificada com ISO 9001:2008, avaliada nível 2 do CMMI-DEV e nível F do MR-MPS-SW. No momento do estudo, a empresa preparava-se para ser avaliada no nível 3 do CMMI-DEV e nível C do MRMPS-SW. A empresa comercializava, além dos seus serviços, produtos considerados COTS $^{2}$.

Para o estudo de viabilidade, a empresa selecionou o processo "Atendimento de Chamados", pois era executado no projeto com maior carteira de clientes. Além disso, a base de métricas da organização era confiável e consistente, o que permitiu analisar o desempenho do processo e definir objetivos de melhoria com métodos quantitativos.

\subsection{Condições do Estudo}

A seleção dos participantes do estudo foi feita por conveniência. O único critério utilizado foi a participação de executores do processo no momento da utilização dos PR. Dois executores do processo foram selecionados. Um executor membro da equipe de desenvolvimento e um executor membro do Grupo de Processo, que participou da definição do processo e auxiliava diariamente a sua execução. O membro do Grupo de Processo atuou também como Responsável do Grupo de Processo (RGP), aplicando o processo. $\mathrm{O}$ membro da equipe selecionado tinha mais de dois anos de experiência no produto e no atendimento de chamados, sendo Analista de Negócios e Especialista de Domínio sênior. O RGP possuía mais de 10 anos na área de qualidade da empresa, certificações referentes à gerência de projetos e era implementador MPS credenciado pela SOFTEX, além de ser responsável pela manutenção da ISO 9001 na organização.

As questões do estudo observadas foram: Q1) qual o grau de satisfação do RGP quanto à facilidade de utilização da abordagem; Q2) qual o grau de satisfação do RGP quanto ao tempo de utilização da abordagem; e Q3) qual o grau de satisfação do RGP quanto ao resultado obtido com a aplicação da abordagem.

As variáveis independentes deste estudo de viabilidade estão relacionadas aos participantes do estudo, ao processo selecionado para melhoria e à organização

${ }^{2}$ Commercial Off-The-Shelf-Componentes de software que oferecem um nível de serviço ou funcionalidade 
supracitadas. As variáveis dependentes que se almejava observar eram: i) facilidade da execução do processo da abordagem; e ii) tempo de execução de cada atividade do processo da abordagem proposta.

No tocante à validade interna do estudo, devem-se considerar as ameaças referentes ao desempenho dos participantes influenciável pela observação, à representatividade do número de participantes e à execução parcial do processo. É importante considerar, sobre esta última ameaça, que o processo da abordagem compreende um ciclo de melhoria e que seus resultados efetivos não puderam ser avaliados, pois não foi possível observar em longo prazo se realmente houve melhoria efetiva no desempenho do processo. Contudo, ao que tange a aplicação dos PR, o estudo compreendeu a execução das tarefas da abordagem cuja composição incluía os PR responsáveis pela investigação da oportunidade de melhoria e pela elaboração da estratégia de solução.

Em relação à validade externa, por questão de tempo restrito para a pesquisa e disponibilidade de organizações candidatas, a execução do estudo aconteceu apenas em um cenário de uma organização de software. O cenário é composto, dentre outros aspectos, pelo porte da empresa, o perfil dos funcionários, a capacidade dos processos e sua maturidade em termos de melhoria contínua dos seus processos organizacionais. A amostragem com representatividade baixa, diante das várias possibilidades de aplicação da abordagem nos diferentes cenários de melhoria de processo de software, configura uma restrição à generalização dos resultados obtidos.

Em relação à validade de construção do estudo, a abordagem foi analisada qualitativamente, não utilizando testes estatísticos. Um aspecto importante a ser considerado é que os participantes envolvidos na execução não eram familiarizados com a TR, eximindo assim o vício que beneficiasse a execução com resultados positivos por conta da experiência dos executores.

\subsection{Execução do Estudo Experimental}

O objetivo de melhoria definido pela alta direção da empresa foi: "Aumentar a eficiência do processo de Atendimento de Chamado". O fator percentual de melhoria da eficiência era definido de acordo com as políticas e diretrizes da organização.

A Figura 4 apresenta o diagrama causal, resultado da aplicação dos procedimentos da ARA, mapeando os efeitos e causas do processo sob o ponto de vista do objetivo de melhoria. Além do diagrama, os procedimentos da ARA, utilizados na atividade "Identificar restrição principal" do processo, mapeia as restrições do processo em termos de grau de impacto. A Tabela 2 apresenta uma amostra da matriz de rastreabilidade das restrições candidatas, juntamente com os efeitos indesejáveis, diretos e indiretos, e o seu grau de impacto. O grau de impacto é calculado com base no número de fatores de influência alcançados direta e indiretamente para cada fator de influência. Desta forma, por exemplo, a restrição candidata de número 14 atingiu 8 de 14 fatores, totalizando $57,14 \%$.

Neste estudo, a restrição identificada como maior limitante foi a "Falta de planejamento da alta direção". A organização sofreu em 2011 um crescimento muito acelerado e isto impactou o tempo de entrega dos seus serviços e produtos. No entanto, embora identificada como candidata a restrição principal, a causa-raiz da restrição, que é a falta de planejamento estratégico organizacional, não poderia ser tratada, a fim de 
que os seus efeitos atuais fossem dirimidos em tempo hábil. A solução de longo prazo para elaborar o planejamento estratégico já estava em andamento na empresa.

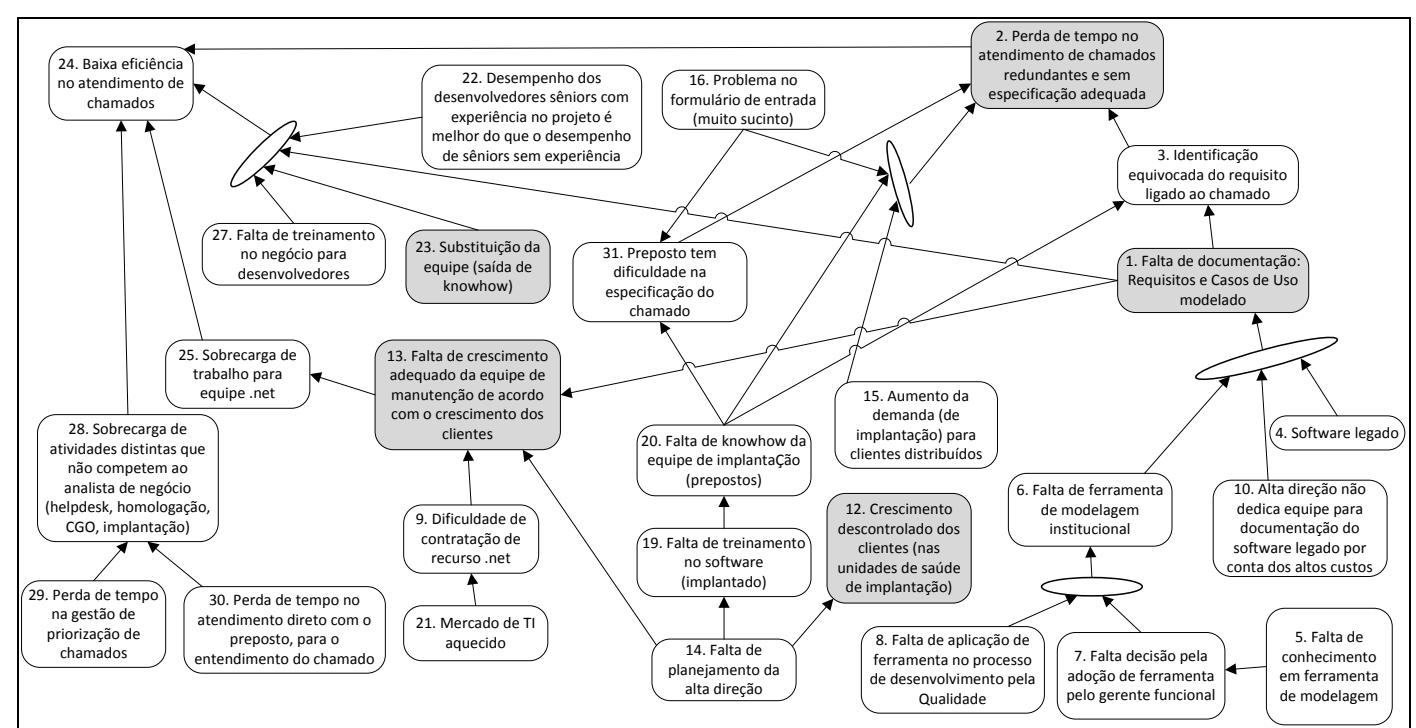

Figura 4 - Diagrama Causal resultado da aplicação dos passos da ARA

A segunda restrição de maior impacto, "Falta de conhecimento em ferramenta de modelagem", foi selecionada como restrição principal. A solução proposta pelo RGP para esta restrição foi adotar e institucionalizar uma ferramenta para modelagem. Não havia nenhum conflito que impedisse a implementação da solução proposta. Por esta razão, a atividade "Elaborar proposta de solução", que incorpora os procedimentos do DRC, foi executada sem a necessidade de utilizar o PR, como prevista no processo.

Após a definição da proposta de solução, a tarefa "Avaliar viabilidade da proposta de solução" foi executada, identificando os efeitos de influência desejáveis e riscos. A Figura 5 apresenta uma parte do diagrama gerado a partir da aplicação dos procedimentos da ARF, contendo o objetivo da proposta de solução, os efeitos desejáveis e as ações que devem ser tomadas para causar tais efeitos.

Tabela 2 - Amostra da matriz de rastreabilidade das restrições candidatas

\begin{tabular}{|c|c|c|c|c|c|c|c|c|c|c|c|c|c|c|c|}
\hline Restrição (fator de influência) & 1 & 2 & 3 & 6 & 7 & 9 & 12 & 13 & 19 & 20 & 24 & 25 & 28 & 31 & $\begin{array}{l}\text { Grau de } \\
\text { impacto } \\
\end{array}$ \\
\hline 21.Mercado de TI aquecido & & & & & & $\mathrm{x}$ & & $\mathrm{x}$ & & & $\mathrm{x}$ & $\mathrm{x}$ & & & $28,57 \%$ \\
\hline 14.Falta de planejamento da alta direção & & $\mathbf{x}$ & $\mathbf{x}$ & & & & $\mathbf{x}$ & $\mathbf{x}$ & $\mathbf{x}$ & $\mathbf{x}$ & $\mathbf{x}$ & $\mathbf{x}$ & & & $57,14 \%$ \\
\hline 04.Software legado & $\mathrm{x}$ & $\mathrm{x}$ & $\mathrm{x}$ & & & & & & & & $\mathrm{x}$ & & & & $28,57 \%$ \\
\hline $\begin{array}{l}\text { 08.Falta de aplicação da ferramenta no processo de } \\
\text { desenvolvimento pela qualidade }\end{array}$ & $\mathrm{x}$ & $\mathrm{x}$ & $\mathrm{x}$ & $\mathrm{x}$ & & & & & & & $\mathrm{x}$ & & & & $35,71 \%$ \\
\hline 05.Falta de conhecimento em ferramenta de modelagem & $\mathbf{x}$ & $\mathbf{x}$ & $\mathbf{x}$ & $\mathbf{x}$ & $\mathbf{x}$ & & & & & & $\mathbf{x}$ & & $\mathbf{x}$ & & $50,00 \%$ \\
\hline $\begin{array}{l}\text { 10.Alta direção não dedica equipe para a documentação do } \\
\text { software legado por conta dos altos custos }\end{array}$ & $\mathrm{x}$ & $\mathrm{x}$ & $\mathrm{x}$ & & & & & & & & $\mathrm{x}$ & & & & $28,57 \%$ \\
\hline 23.Substituição da equipe (saída de know-how) & & & & & & & & & & & $\mathrm{x}$ & & & & $7,14 \%$ \\
\hline 16.Problema no formulário de entrada (muito sucinto) & & $\mathrm{x}$ & & & & & & & & & $\mathrm{x}$ & & & $\mathrm{x}$ & $21,43 \%$ \\
\hline $\begin{array}{l}\text { 15. Aumento de demanda (de implantação) para clientes } \\
\text { distribuídos }\end{array}$ & & $\mathrm{x}$ & & & & & & & & & $\mathrm{x}$ & & & & $14,29 \%$ \\
\hline $\begin{array}{l}\text { 30. Perda de tempo no atendimento direto com o preposto, } \\
\text { para o atendimento do chamado }\end{array}$ & & & & & & & & & & & $\mathrm{x}$ & & $\mathrm{x}$ & & $14,29 \%$ \\
\hline
\end{tabular}




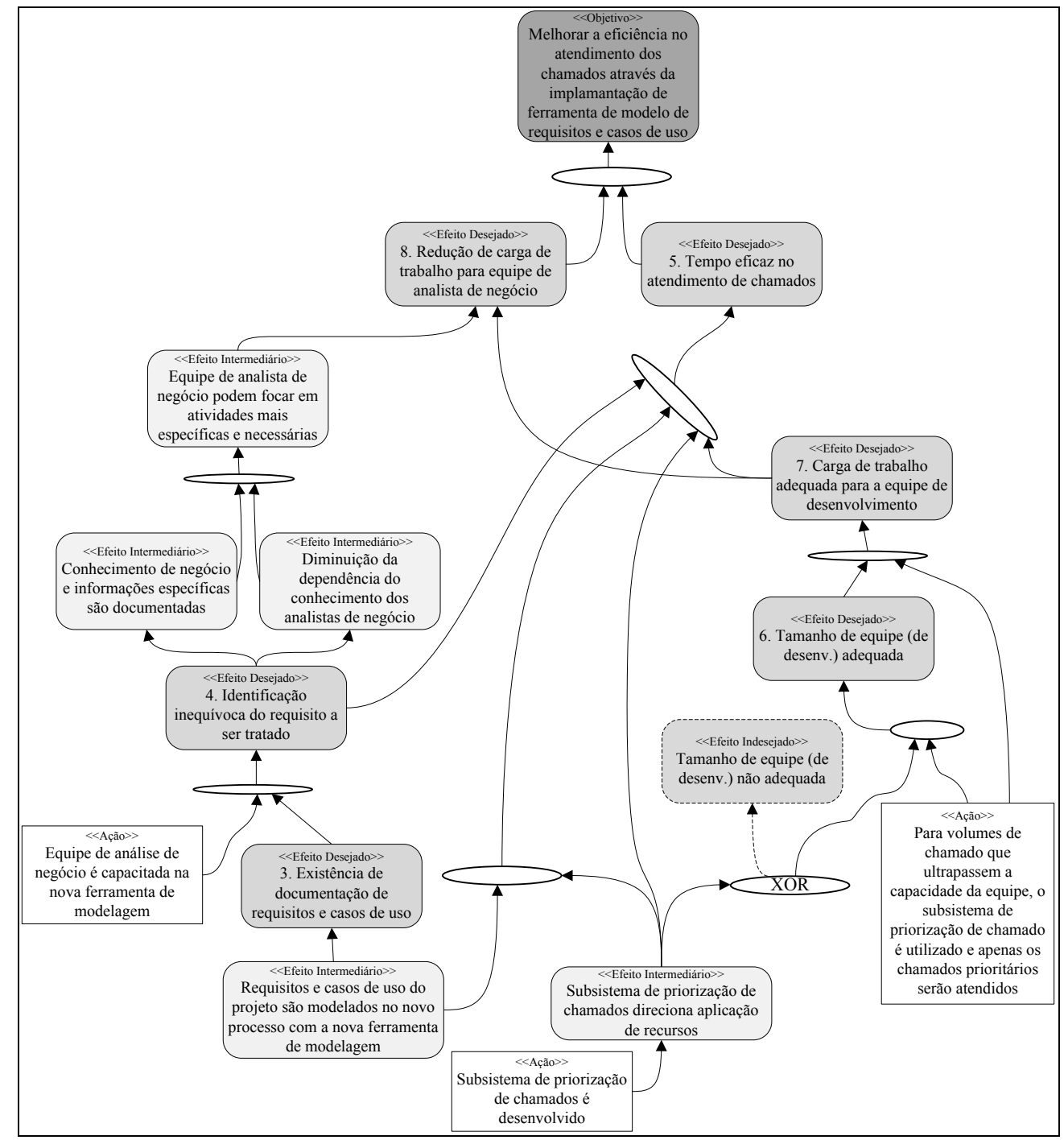

Figura 5 - Amostra da Análise de Viabilidade da Proposta de Solução (ARF)

\subsection{Análise da Execução do Estudo}

Além do acompanhamento do tempo de execução e de observações coletadas pelo pesquisador ao longo da execução do estudo, os participantes preencheram um formulário ao final do estudo respondendo sobre as percepções que se desejava observar de acordo com as questões de pesquisa. Informações detalhadas e formulários preenchidos podem ser encontrados em (Costa 2012).

No tocante a questão de pesquisa Q1, os participantes conseguiram executar os procedimentos dos PR. A percepção dos participantes apontou uma dificuldade inicial no entendimento dos procedimentos dos PR. Segundo o RGP, embora os conceitos da TR sejam simples, a execução dos procedimentos dos PR não é trivial inicialmente. Os modelos de formulário providos juntos ao processo também contribuíram para a sua execução. Um treinamento ou material de apoio para apoiar a apresentação dos procedimentos dos PR aos participantes poderia suplantar tal dificuldade. 
Em relação à questão Q2, o tempo total de execução do processo foi de 10 horas e 36 minutos. De acordo com o RGP, para um processo que considera desde a definição de objetivos de melhoria até a definição da proposta de melhoria, o tempo consumido na execução da abordagem é razoável para a realidade da empresa. No entanto, foi observado pelo RGP que a sistemática da abordagem é exaustiva, mas que era justificada pela importância da melhoria daquele processo crítico. Esta percepção indica que a abordagem proposta é bem aproveitada em situações nas quais o processo já está otimizado e constitui um processo crítico para a organização.

Sobre a questão Q3, o RGP argumentou que, embora houvesse suspeitas sobre a restrição principal do desempenho do processo, não havia nenhum consenso dos envolvidos ou constatação a respeito. Dessa forma, a abordagem permitiu identificar a restrição principal de forma sistemática, corroborando com a suspeita dos executores do processo.

Desta forma, é possível afirmar que há indícios de aplicabilidade dos PR à melhoria de processo de software. Além disso, há indicativos de que a aplicação da abordagem também seja viável em termos de tempo de execução.

\section{Conclusão}

Processos que estão em melhoria contínua tendem a ficarem mais difíceis de serem otimizados, pois problemas na sua execução e suas recorrências são tratados ao longo do tempo, diminuindo assim as variações por causas especiais no seu desempenho. Além disso, processos de software são peculiares, pois a sua execução, na maioria das vezes, produz produtos diferentes e se baseia fortemente no esforço intelectual e colaborativo dos envolvidos (Lindvall e Rus 2000).

Um mapeamento sistemático realizado para identificar abordagens para melhoria contínua de processo de software em organizações cujos processos fossem maduros ou estivessem sob controle estatístico confirmou a necessidade por uma abordagem que apoiasse a melhoria contínua de processos de software de maneira sistemática desde a identificação da oportunidade de melhoria até a proposição e implementação da melhoria.

A Teoria das Restrições é uma abordagem de melhoria contínua de processo que considera as causas e efeitos dos fatores de influência do processo para identificar a oportunidade de melhoria (restrição principal), para avaliar a viabilidade da proposta de solução e para elaborar a proposta de melhoria. A similaridade, convergência e composição dos PR com outras abordagens de TQM, que já haviam sido aplicadas com sucesso na melhoria de processo de software, tais como, Seis Sigma e Controle Estatístico de Processo, serviram como hipótese inicial da aplicabilidade da TR à melhoria de processo de software. Mais informações sobre a aplicação da TR nos diversos campos e sua combinação com outras abordagens de TQM podem ser encontradas em (Costa 2012).

Um estudo experimental realizado em uma organização de software para averiguar a aplicabilidade dos PR para melhoria de processo de software apontou indícios de aplicabilidade e de viabilidade. No estudo, os resultados obtidos com a aplicação do processo foram condizentes com as suspeitas dos responsáveis do Grupo de Processo. O tempo de execução da parte da investigação da oportunidade de melhoria e da proposição da solução foi de 10 horas e 36 minutos, sendo considerado 
plausível, tendo em vista os benefícios, a certificação da oportunidade de melhoria e os produtos gerados com a execução do processo (proposta de melhoria, produtos correlatos e lições aprendidas).

Outros estudos experimentais se fazem necessários para avaliar a abordagem em cenários mais diversos, como, por exemplo, em organizações de alta maturidade com foco em processos sob controle estatístico, pois permitiria um melhor aproveitamento do rigor proposto pelos PR. O desenvolvimento de uma ferramenta que apoie a integração de todo o processo é oportuna, relacionando os objetivos organizacionais, objetivos de desempenho dos processos, a execução dos procedimentos dos PR e a integração dos resultados às bases de melhoria e de lições aprendidas da organização.

No tocante às contribuições, considerando os indícios de aplicabilidade dos PR à melhoria de processo de software, podem ser citadas as seguintes: i) apoio à identificação da restrição principal do desempenho do processo (causa-raiz); ii) apoio à resolução de conflitos relacionados à solução para melhoria de processos de software; iii) apoio à elaboração de proposta de solução para remover restrições no desempenho do processo; iv) apoio à identificação de riscos relacionados à melhoria do processo; v) definição de um processo de melhoria contínua de processo de software; vi) aplicação dos procedimentos dos Processos de Raciocínio à melhoria de processos de software com base nos conceitos da Teoria das Restrições; vii) apoio à melhoria de processos por meio de formulários que estruturam as informações e o raciocínio utilizado ao longo do processo de investigação e elaboração da solução e melhoria; e vii) processo e formulários que observam práticas e resultados esperados preconizados por modelos de maturidade, tais como, MR-MPS-SW e CMMI-DEV, no que tange o ciclo de melhoria contínua.

\section{Bibliografia}

Albuquerque, A. B. (2008). Avaliação e Melhoria de Ativos de Processos Organizacionais em Ambientes de Desenvolvimento de Software. Rio de Janeiro, Universidade Federal do Rio de Janeiro.

Campos, F. B. (2008). Avaliação e Melhoria de Ativos de Processos de Software de Instituições Implementadoras. COPPE. Rio de Janeiro, UFRJ. D. Sc.: 305.

Costa, T. M. d. (2012). Melhoria Contínua de Processo de Software Utilizando a Teoria das Restrições. Programa de Engenharia de Sistemas e Computação. Rio de Janeiro, UFRJ. M.Sc.

Davies, J., V. Mabin, et al. (2005). "The theory of constraints: a methodology apart?--a comparison with selected OR/MS methodologies." Omega 33(6): 506-524.

Davies, J., V. Mabin, et al. (2004). The theory of constraints and systems dynamics: A suitable case for multimethodology.

Ehie, I. and C. Sheu (2005). "Integrating six sigma and theory of constraints for continuous improvement: a case study." Journal of Manufacturing Technology Management 16(5): 542-553.

Florac, W. and A. Carleton (1999). Measuring the software process: statistical process control for software process improvement, Addison-Wesley Longman Publishing Co., Inc. Boston, MA, USA. 
Goldratt, E. M. (1990). What Is This Thing Called the Theory of Constraints and How Should It Be Implemented? Croton-on-Hudson, NY, North River Press.

Gupta, M. C. and L. H. Boyd (2008). "Theory of constraints: a theory for operations management." International Journal of Operations \& Production Management 28(10): 991-1012.

Hong, G. Y. and T. N. Goh (2003). "Six Sigma in software quality." TQM Magazine 15(6): 364-373.

ISO/IEC (2003). ISO/IEC 15504: Information Technology - Software Process Assessment, The International Organization for the Standardization and the International Electrotechnical Commission. 15504.

ISO/IEC (2008). ISO/IEC 12207: System and Software Engineering - Software Life Cycle Processes, The International Organization for the Standardization and the International Electrotechnical Commission.

Lepore, D. and O. Cohen (1999). Deming and Goldratt: The Theory of Constraints and the System of Profound Knowledge. Great Barrington, MA, North River Press.

Lindvall, M. and I. Rus (2000). "Process diversity in software development." IEEE software 17(4): 14-18.

Montoni, M. (2010). Uma Investigação sobre os Fatores Críticos de Sucesso em Iniciativas de Melhoria de Processos de Software. Rio de Janeiro, Universidade Federal do Rio de Janeiro, COPPE.

Nave, D. (2002). "How to Compare Six Sigma, Lean and the Theory of Constraints." Quality Progress 35(3): 73.

Pirasteh, R. and K. Farah (2006). "Continuous Improvement Trio: Combining the theory of constraints, lean, and six sigma to form" TLS" is an innovative process improvement methodology with real-world results." Apics - The Performance Advantage 16(5): 31.

Scheinkopf, L. J. (1999). Thinking for a change: Putting the TOC thinking processes to use. Boca Raton, FL, CRC Press.

SEI (2010). CMMI ${ }^{\circledR}$ for Development (CMMI-DEV), V1.3, CMU/SEI-2010. Software Engineering Institute. Disponível em: http://www.sei.cmu.edu/.

Silva Filho, R. C. (2006). Uma Abordagem para Avaliação de Propostas de Melhoria em Processos de Software. Engenharia de Sistemas e Computação. Rio de Janeiro, Universidade Federal do Rio de Janeiro. M.Sc.: 167.

SOFTEX (2012). MPS.BR - Melhoria de Processo do Software Brasileiro - Guia Geral. http://www.softex.br/mpsbr.

Watson, K., J. Blackstone, et al. (2007). "The evolution of a management philosophy: the theory of constraints." Journal of operations management 25(2): 387-402. 